\title{
ON NORM CONTINUITY, DIFFERENTIABILITY AND COMPACTNESS OF PERTURBED SEMIGROUPS
}

\author{
A. BOULOUZ, H. BOUNIT, A. DRIOUICH AND S. HADD
}

\begin{abstract}
The main purpose of this paper is to treat semigroups properties, like norm continuity, compactness and differentiability for perturbed semigroups in Banach spaces. In particular, we investigate three large classes of perturbations, Miyadera-Voigt, DeschSchappacher and Staffans-Weiss perturbations. Our approach is mainly based on feedback theory of Salamon-Weiss systems. Our results are applied to abstract boundary integro-differential equations in Banach spaces.
\end{abstract}

\section{INTRODUCTION}

In this paper, we investigate classical properties like norm continuity, compactness and differentiability for some classes of perturbed semigroups. To be more precise, let Banach spaces $X, Z$ such that $Z \subset X,(A, D(A))$ a generator of a strongly continuous semigroup $\mathbb{T}:=(T(t))_{t \geq 0}$ on $X$ such that $D(A) \subset Z$ and a linear operator $\mathbb{L} \in \mathcal{L}(\tilde{Z}, \tilde{X})$ with $\tilde{Z}$ and $\tilde{X}$ are Banach spaces carefully chosen in order that $A+\mathbb{L}$ with appropriate domain is well defined and generates a strongly continuous semigroup $\mathbb{T}^{c l}:=\left(T^{c l}(t)\right)_{\geq 0}$ on $X$ (this notation will be justified in the next section). Now the problem to be treated is: does semigroups generated by $A$ and $A+\mathbb{L}$ permute the aforementioned properties? As a matter of facts this problem is already considered by many authors and have some partial answers (depending on the type of perturbations).

The class of bounded perturbations, i.e. the case when $X=\tilde{Z}=\tilde{X}$ (so that $\mathbb{L} \in \mathcal{L}(X)$ ) is mainly treated by Phillips [22. He proved that if $(T(t))_{t \geq 0}$ is norm continuous (resp. compact) for $t>0$, then the operator $(A+\mathbb{L}, D(A))$ generates a strongly continuous semigroup $\left(T^{c l}(t)\right)_{t \geq 0}$ on $X$ is norm continuous (resp. compact) for $t>0$, as well. On the other hand, Phillips constructed a semigroup $(T(t))_{t \geq 0}$ which is norm continuous for $t>t_{0}$ with $t_{0}>0$ (i.e. eventually norm continuous) but the semigroup $\left(T^{c l}(t)\right)_{t \geq 0}$ is not norm continuous for $t>t_{0}$. Thus eventual norm continuity and eventual compactness are, in general, not preserved under even bounded perturbations. It is shown in [7] that in the case of compact perturbation operator $\mathbb{L} \in \mathcal{L}(X)$, the eventual norm continuity is preserved for the perturbed semigroup whenever the initial semigroup is. In 1983, Pazy [21] (see also [23]) showed that the eventual differentiability of the semigroup $(T(t))_{t \geq 0}$ is not translated to the perturbed semigroup generated by $A+\mathbb{L}$ even if $\mathbb{L}$ is a bounded perturbation. As shown in [2], 3], 6], [13], and [32, extra conditions on the semigroup

Key words and phrases. Operator semigroup, unbounded perturbation, norm continuity, compactness, differentiability, Bergman space, feedback theory, integro-differential equations. 
$\mathbb{T}$ are needed to assure the preservation of the eventual differentiability for the semigroup generated by $A+\mathbb{L}$.

Let us now analysing in profile the case of unbounded perturbations. Three large classes of unbounded perturbations will be investigated. The first class: choose $\tilde{Z}=Z$ and $\tilde{X}=X$, then we say that $\mathbb{L} \in \mathcal{L}(Z, X)$ is a Miyadera-Voigt perturbation for $A$ if there exist $\alpha>0$ and $\gamma \in(0,1)$ such that for any $x \in D(A)$ we have

$$
\int_{0}^{\alpha}\|\mathbb{L} T(t) x\| d t \leq \gamma\|x\| .
$$

In this case the operator $(A+\mathbb{L}, D(A))$ generates a strongly continuous semigroup $\left(\mathbb{T}^{c l}(t)\right)_{t \geq 0}$ on $X$. As an example of application we cite the case of delay equations [1], [11. In general, we do not have preservation of the aforementioned regularities under Miyadera-Voigt perturbations. However, for delay evolution equations with $L^{p}$-history spaces it show in [1] (resp. [18]) that if the free delay equation is governed by an immediately norm continuous semigroup (resp. immediately compact), then the delay semigroup in product spaces associated with the delay equation is eventually norm continuous (resp. eventually compact). Moreover, some results on eventual differentiability for delay equations is obtained in [3. The second class of unbounded perturbations is the case $\tilde{Z}=X$ and $\tilde{X}=X_{-1}$, so that $\mathbb{L} \in \mathcal{L}\left(X, X_{-1}\right)$, where $X_{-1}$ is the completion of $X$ with respect to the norm $\|x\|_{-1}:=\left\|(\lambda I-A)^{-1} x\right\|, x \in X$. The semigroup $\mathbb{T}$ can be extended to a strongly continuous semigroup $\mathbb{T}_{-1}:=\left(T_{-1}(t)\right)_{t \geq 0}$ on $X_{-1}$, whose generator $A_{-1}: X \rightarrow X_{-1}$, the extension of $A$ to $X$. In this case we say that $\mathbb{L}$ is a Desch-Schappacher perturbation for $A$ if there exists $t_{0}>0$ such that

$$
\int_{0}^{t_{0}} T_{-1}\left(t_{0}-s\right) \mathbb{L} f(s) d s \in X, \quad \forall f \in L^{1}\left(\left[0, t_{0}\right], X\right) .
$$

It is well-know (see [7, chapter III-3-d]) that for a such $\mathbb{L}$, the part of the operator $A_{-1}+\mathbb{L}$ on $X$ (denoted by $\left.A^{c l}:=\left(A_{-1}+\mathbb{L}\right)_{\mid X}\right)$ generates a strongly continuous semigroup $\mathbb{T}^{c l}$ on $X$. For this kind of perturbations, Mátrai [19] has showed that $\mathbb{T}^{c l}$ is immediately normcontinuous whenever the semigroup $\mathbb{T}$ is (see also Jung [16]). To the best of our knowledge, there is no results concerning the differentiability under Desch-Schappacher perturbations. This is one of the objectives of this paper. In fact, we will show that if the generator $A$ satisfies the so-called the Pazy condition [21, p.57] (see also (4.1) below), then the semigroup generated by $A^{c l}$ is differentiable. Finally, let us discuss another more general class of perturbations. To that purpose, let $A_{m}: Z \subset X \rightarrow X$ be a differential linear closed operator and $G, M: Z \rightarrow U$, boundary linear operators, where $U$ is a (boundary) Banach space. We consider the linear operator on $X$,

$$
\mathcal{A}:=A_{m}, \quad D(\mathcal{A})=\{f \in Z, \quad G f=M f\} .
$$

We assume that $A:=A_{m}$ with domain $D(A)=\operatorname{ker}(G)$ is a generator of a strongly continuous semigroup $\mathbb{T}$ on $X$. Observe that the operator $\mathcal{A}$ is obtained by perturbing the domain $D(A)$ of $A$ by an unbounded perturbation $M$. Based on feedback theory of regular linear systems ([30], see also the next section for definitions), the authors of 
[12] introduced sufficient conditions on $G$ and $M$ for which $(\mathcal{A}, D(\mathcal{A}))$ is a generator of a strongly continuous semigroup $\mathbb{T}^{c l}$ on $X$. In fact, they proved that there exists a space $\tilde{Z}$ such that $D(A) \subsetneq Z \subset \tilde{Z} \subsetneq X$ and an operator $\mathbb{L} \in \mathcal{L}\left(\tilde{Z}, X_{-1}\right)$ such that the operator $(\mathcal{A}, D(\mathcal{A}))$ coincides with the following one

$$
A^{c l}:=A_{-1}+\mathbb{L}, \quad D\left(A^{c l}\right)=\left\{x \in \tilde{Z}:\left(A_{-1}+\mathbb{L}\right) x \in X\right\} .
$$

In this case, the operator $\mathbb{L} \in \mathcal{L}\left(\tilde{Z}, X_{-1}\right)$ is called a Staffans-Weiss perturbation (see Theorem 2.8). The main objective of this work is to prove that immediate norm continuity and compactness are preserved under Staffans-Weiss perturbations operators, see Theorem 3.3 and Theorem 3.4 below. Particular case of these results is when the operator $M$ is bounded, i.e. $M \in \mathcal{L}(X)$, so that we are in Desch-Schappacher perturbations setting.

As source of applications of our abstract results, we will consider regularity of solutions of the following intergo-differential equation

$$
\begin{cases}\dot{x}(t)=A_{m} x(t)+\int_{0}^{t} k(t-s) P x(s) d s, & t \geq 0 \\ G x(t)=M x(t), & t \geq 0, \\ x(0)=x, & \end{cases}
$$

where $A_{m}, G$ and $M$ as above, $P: Z \rightarrow X$ is an admissible observation operator for $A$, and the kernel $k(\cdot)$ belong to an appropriate Bergman space (see Section 5).

For the reader's convenience, we briefly recall the relevant background from [30] (and also [12]) and related works and introduce (much of) our notation in Section 2, Section 3 is on the study of immediate norm continuity and compactness of semigroups under StaffansWeiss perturbations operators. In section 4 we investigate the eventual differentiability of semigroups under Desch-Schappacher perturbations. The last section is concerned with the study of a class of integro-differential equations in Banach spaces.

\section{Staffans-Weiss PERTURBAtion THEOREM}

In this section, we shall recall the recent concept of Staffans-Weiss perturbations. The origin of these perturbations is the feedback theory of well-posed and regular linear systems introduced mainly by Salamon, Staffans and Weiss, see e.g. [24], 26, chap.7] and 30.

Throughout this section $X$ and $U$ are Banach spaces (with norms denoted (for simplicity) by the same symbol $\|\cdot\|)$ and $p>1$ is a real number. Let $Z$ be another Banach space such that $Z \subset X$ (with continuous and dense embedding). We now consider a differential operator $A_{m}: Z \rightarrow X$ and a trace operator $G: Z \rightarrow U$ assumed to be surjective. We also assume that the following operator

$$
A=A_{m} \quad D(A)=\{x \in Z: G x=0\}
$$

generates a strongly continuous semigroup $\mathbb{T}:=(T(t))_{t \geq 0}$ on $X$ of type $\omega_{0}(A)$. We denote by $\rho(A)$ the resolvent set of $A, \sigma(A)=\mathbb{C} \backslash \rho(A)$ the spectrum of $A$, and $R(\lambda, A):=$ $(\lambda I-A)^{-1}$ for $\lambda \in \rho(A)$, the resolvent operator of $A$. The graph norm with respect to 
$A$ is $\|x\|_{A}:=\|x\|+\|A x\|$ for $x \in D(A)$. It is well-known that $X_{A}:=\left(D(A),\|\cdot\|_{A}\right)$ is a Banach space and $X_{A} \hookrightarrow X$ (densely and continuously). It is shown by Greiner [10] that the restriction of $G$ to $\operatorname{ker}\left(\lambda-A_{m}\right)$ for $\lambda \in \rho(A)$ is invertible. As known, the inverse

$$
D_{\lambda}:=\left(G_{\mid \operatorname{ker}\left(\lambda-A_{m}\right)}\right)^{-1} \in \mathcal{L}(U, Z)
$$

is called the Dirichlet operator. On the other hand, we define a new norm on $X$ by setting $\|x\|_{-1}:=\|R(\beta, A) x\|$ for $x \in X$ and some (hence all) $\beta \in \rho(A)$. The completion of $X$ with respect to this norm is a Banach space denoted by $X_{-1}$ and satisfies

$$
X_{A} \hookrightarrow X \hookrightarrow X_{-1} .
$$

The extension of the semigroup $\mathbb{T}$ to $X_{-1}$ is again a strongly continuous semigroup $\mathbb{T}_{-1}:=$ $\left(T_{-1}(t)\right)_{t \geq 0}$ on $X_{-1}$ whose generator $A_{-1}: D\left(A_{-1}\right)=X \rightarrow X_{-1}$ is the extension of $A$ to $X$. We consider the boundary control problem

$$
\left\{\begin{array}{l}
\dot{z}(t)=A_{m} z(t), \quad t \geq 0, \\
z(0)=x, \\
G z(t)=u(t), \quad t \geq 0,
\end{array}\right.
$$

where $u:[0,+\infty) \rightarrow U$ is a control function (boundary control). If one looks for a solution $z \in C([0, \alpha], X)$ (with $\alpha>0$ ) of (2.3), it is more convenient to reformulate a such boundary problem as a distributed one. To that purpose, we introduce the following operator

$$
B:=\left(\lambda-A_{-1}\right) D_{\lambda} \in \mathcal{L}\left(U, X_{-1}\right), \quad \lambda \in \rho(A),
$$

(this operator do not depend of $\lambda$, due to the resolvent equation). One can see that the boundary problem (2.3) is equivalent to

$$
\left\{\begin{array}{l}
\dot{z}(t)=A_{-1} z(t)+B u(t), \quad t \geq 0 \\
z(0)=x
\end{array}\right.
$$

The solution of (2.5) (and hence of (2.3)) is given by

$$
\begin{aligned}
z(t) & =T(t) x+\int_{0}^{t} T_{-1}(t-s) B u(s) d s \\
& :=T(t) x+\Phi_{t} u,
\end{aligned}
$$

for any $t \geq 0, x \in X$ and $u \in L^{p}([0,+\infty), U)$. We have

$$
\Phi_{t} \in \mathcal{L}\left(L^{p}([0, t], U), X_{-1}\right), \quad \forall t>0 .
$$

Observe that the solution $z$ takes value in $X_{-1}$. We then have the following definition.

Definition 2.1. The operator $B \in \mathcal{L}\left(U, X_{-1}\right)$ is called an admissible control operator for $A$ if there exists $t_{0}>0$ such that Range $\left(\Phi_{t_{0}}\right) \subset X$. In this case we also say that the pair $(A, B)$ is admissible or sometimes well-posed. 
If $(A, B)$ is well-posed then we have $\Phi_{t} \in \mathcal{L}\left(L^{p}([0, t], U), X\right)$ for any $t>0$ and the solution of (2.3) satisfies $z \in C\left(\mathbb{R}^{+}, X\right)$, see [29], [27, chap.4]. The familly $\left(\Phi_{t}\right)_{t \geq 0}$, satisfies for all $t, \tau \geq 0$,

$$
\Phi_{t+\tau} u=T(t) \Phi_{\tau}\left(u_{\mid[0, \tau]}\right)+\Phi_{t} u(\cdot+\tau)
$$

for any $u \in L^{p}([0, t+\tau], U)$. In addition

$$
\left\|\Phi_{\tau_{1}}\right\| \leq\left\|\Phi_{\tau_{2}}\right\|
$$

for any $0 \leq \tau_{1} \leq \tau_{2}$. Moreover, for all $\omega>\omega_{0}(A)$, there exists a constant $c>0$ such that

$$
\left\|R\left(\lambda, A_{-1}\right) B\right\| \leq \frac{c}{(\operatorname{Re} \lambda-\omega)^{\frac{1}{p}}}
$$

for any $\lambda \in \mathbb{C}$ such that $\operatorname{Re} \lambda>\omega$.

Let us now consider the following observed boundary system

$$
\begin{cases}\dot{z}(t)=A_{m} z(t), & t \geq 0 \\ z(0)=x, & \\ G z(t)=0, & t \geq 0 \\ y(t)=M z(t), & t \geq 0\end{cases}
$$

where $M: D(M)=Z \rightarrow U$ is a linear operator and $y:[0,+\infty) \rightarrow U$ is an observation function. The problem is how to extend the observation function $y(\cdot ; x)$ to a function in $L_{l o c}^{p}\left(\mathbb{R}^{+}, U\right)$ for any initial condition $x \in X$. To that purpose, let $(A, D(A))$ be defined by (2.1) and select

$$
C=M \imath \in \mathcal{L}\left(X_{A}, U\right)
$$

where $\imath: D(A) \rightarrow Z$ is the continuous injection. Then the system (2.10) is reformulated as

$$
\left\{\begin{array}{l}
\dot{z}(t)=A z(t), \quad t \geq 0, \\
z(0)=x, \\
y(t)=C z(t), \quad t \geq 0 .
\end{array}\right.
$$

The mild solution of the evolution equation in (2.12) is given by $z(t)=T(t) x$ for any $t \geq 0$ and $x \in X$. However the observation $y(t)$ is only defined on the domain $D(A)$ and we have $y(t)=C T(t) x$ for any $t \geq 0$ and initial condition $x \in D(A)$. We need the following definition

Definition 2.2. $C \in \mathcal{L}(D(A), U)$ is called an admissible observation operator for $A$ (we also say that $(C, A)$ is admissible or well-posed) if

$$
\int_{0}^{\tau}\|C T(s) x\|^{p} d s \leq \gamma^{p}(\tau)\|x\|^{p}
$$

for all $x \in D(A)$ and for some constants $\tau>0$ and $\gamma:=\gamma(\tau)>0$. 
To state our main results in next sections, we need to define the concept of zero class admissible observation operators which was first introduced in [33], in order to provide conditions for exact observability of semigroup systems. This concept was further developed in [15].

Definition 2.3. The operator $C \in \mathcal{L}(D(A), U)$ is said to belong to the zero class of admissible observation operators for $A$ ( $C$ is zero-class admissible), if the best constant $\gamma(\tau)$, given by (2.13), satisfies $\gamma(\tau) \rightarrow 0$ as $\tau \rightarrow 0$.

Obviously, bounded observation operators $C \in \mathcal{L}(X, U)$ are zero-class admissible. Let $(C, A)$ be admissible. Due to (2.13) and the density of the domain $D(A)$ in $X$, the linear operator

$$
\Psi_{\infty}: D(A) \rightarrow L_{l o c}^{p}([0,+\infty), U), \quad x \mapsto \Psi_{\infty} x=C T(\cdot) x
$$

is extended to a linear bounded operator $\Psi_{\infty}: X \rightarrow L_{l o c}^{p}([0,+\infty), U)$. Then the observation function $y$ can be extended to a $p$-locally integrable function for any initial condition $x \in X$ by setting

$$
y(t)=\left(\Psi_{\infty} x\right)(t), \quad \text { a.e. } t \geq 0
$$

Next we recall the representation of $\Psi_{\infty}$ (then $y$ ) using an extension operator of $C$. We then need the following concept.

Definition 2.4. The Yosida extension of an operator $C \in \mathcal{L}(D(A), U)$ with respect to $A$ is the operator defined by

$$
\begin{aligned}
C_{\Lambda} x & :=\lim _{\lambda \rightarrow+\infty} C \lambda R(\lambda, A) x \\
D\left(C_{\Lambda}\right) & :=\left\{x \in X, \lim _{\lambda \rightarrow+\infty} C \lambda R(\lambda, A) x \text { exists in } U\right\} .
\end{aligned}
$$

Let $\lambda_{0}$ such that $\left[\lambda_{0}, \infty\right) \subset \rho(A)$. We define the following norm

$$
\|x\|_{D\left(C_{\Lambda}\right)}:=\|x\|_{X}+\sup _{\lambda \geq \lambda_{0}}\|C \lambda R(\lambda, A) x\|_{U}, \quad x \in D\left(C_{\Lambda}\right) .
$$

According to [28], $\left[D\left(C_{\Lambda}\right)\right]:=\left(D\left(C_{\Lambda}\right),\|\cdot\|_{D\left(C_{\Lambda}\right)}\right)$ is a Banach space. Now if $(C, A)$ is admissible, the representation theorem of Weiss (see [28]) shows that Range $(T(t)) \subset$ $D\left(C_{\Lambda}\right)$ for a.e. $t>0$. On the other hand, for any $x \in X$ and a.e. $t \geq 0$, we have

$$
\left(\Psi_{\infty} x\right)(t)=C_{\Lambda} T(t) x
$$

A necessary condition for the well-posedness of $(C, A)$ is: for all $\omega \in \mathbb{C}$ with $\omega>\omega_{0}(A)$, there exists a constant $b>0$ such that

$$
\|C R(\lambda, A)\| \leq \frac{b}{(\operatorname{Re} \lambda-\omega)^{1-\frac{1}{p}}}, \quad \operatorname{Re} \lambda>\omega,
$$

see [26], [27], and [28], for more details. 
Let us now consider the input-output boundary system

$$
\begin{cases}\dot{z}(t)=A_{m} z(t), & t \geq 0 \\ G z(t)=u(t), & t \geq 0 \\ y(t)=M z(t), & t \geq 0 \\ z(0)=x, & \end{cases}
$$

The question now is how to define the well-posedness of the system (2.15) in the sense that the solution $z(t ; x, u)$ should belong to the state space $X$ for any $x \in X, u \in$ $L^{p}([0,+\infty), U)$ and a.e. $t \geq 0$, continuously depends on $t$ and $u$, and the transformation $u \mapsto y$ define a linear bounded operator on $L_{l o c}^{p}([0,+\infty), U)$. Using operators (2.4) and (2.11), the system (2.15) is reformulated as

$$
\begin{cases}\dot{z}(t)=A z(t)+B u(t), & t \geq 0, \\ z(0)=x, & t \geq 0 . \\ y(t)=C z(t), & \end{cases}
$$

We start by assuming that $(A, B)$ and $(C, A)$ are admissible. In this case, $z(t)=T(t) x+$ $\Phi_{t} u \in X$ for any $t \geq 0$ and $u \in L^{p}([0,+\infty), U)$. We now look for additional condition so as to extend the output function $y$ to a function in $L_{l o c}^{p}\left(\mathbb{R}^{+}, U\right)$. For this, define the space

$$
W_{0, l o c}^{2, p}([0,+\infty), U):=\left\{v \in W_{l o c}^{2, p}([0,+\infty), U): v(0)=v^{\prime}(0)=0\right\},
$$

which is dense in $L_{l o c}^{p}([0,+\infty), U)$. Without loss generality, we can assume that $0 \in$ $\rho(A)$, so $B=\left(-A_{-1}\right) D_{0}$ (see (2.4) $)$. Now an integration by parts yields, for any $u \in$ $W_{0, l o c}^{2, p}([0,+\infty), U)$,

$$
\Phi_{t} u=D_{0} u(t)-\int_{0}^{t} T(t-s) D_{0} u^{\prime}(s) d s \in Z, \quad t \geq 0 .
$$

This allows us to define the following operator

$$
\left(\mathbb{F}_{\infty} u\right)(t)=M \Phi_{t} u, \quad u \in W_{0, l o c}^{2, p}([0,+\infty), U) \text {, a.e. } t \geq 0 .
$$

Definition 2.5. The system $(A, B, C)$ is called well-posed on $X, U, U$ if

(1) $(A, B)$ is well-posed on $X, U$,

(2) $(C, A)$ is well-posed on $X, U$, and

(3) there exist constants $\tau>0$ and $\kappa_{\tau}>0$ such that

$$
\left\|\mathbb{F}_{\infty} u\right\|_{L^{p}([0, \tau], U)} \leq \kappa_{\tau}\|u\|_{L^{p}([0, \tau], U)}, \quad u \in W_{0, l o c}^{2, p}([0,+\infty), U) .
$$

The first consequence of the well-posedness of the system $(A, B, C)$ is that the operator $\mathbb{F}_{\infty}$ can be extended to a linear bounded operator on $L_{l o c}^{p}([0,+\infty), U)$, denoted again by $\mathbb{F}_{\infty}$. On the other hand, observation function of the system (2.15) is given by

$$
y=\Psi_{\infty} x+\mathbb{F}_{\infty} u, \quad(x, u) \in X \times L_{l o c}^{p}([0,+\infty), U),
$$

which is a function in $L_{l o c}^{p}([0,+\infty), U)$. Observe that the Laplace transform of $\mathbb{F}_{\infty}$ is given by

$$
\widehat{\left(\mathbb{F}_{\infty} u\right)}(\lambda)=M R\left(\lambda, A_{-1}\right) B \hat{u}(\lambda)
$$


for $\lambda \in \rho(A)$ such that these Laplace transforms exist.

Now in order to given a representation of the observation function $y(t)$ is terms of the operator $C$ and the state $z(t)$ of the system (2.15), we need the following important subclass of well-posed systems.

Definition 2.6. A well-posed system $(A, B, C)$ is regular on $X, U, U$ (with feedthrough $D=0)$ if

$$
\lim _{t \rightarrow 0^{+}} \frac{1}{t} \int_{0}^{t}\left(\mathbb{F}_{\infty} u_{0}\right)(\sigma) d \sigma=0
$$

exists in $U$, for the constant control function $u_{0}(t)=v, v \in U, t \geq 0$.

If the system $(A, B, C)$ is regular and by using a tauberian theorem we have

$$
\lim _{\lambda>0, \lambda \rightarrow+\infty} \lambda\left(\widehat{\mathbb{F}_{\infty} u_{0}}\right)(\lambda)=0 .
$$

According to (2.18), if $(A, B, C)$ is regular then

$$
\lim _{\lambda \rightarrow+\infty} M R\left(\lambda, A_{-1}\right) B v=0, \quad v \in U .
$$

In addition, if we take $\lambda>0$ sufficiently large and $\mu \in \rho(A)$, then by using (2.19), and the following identity

$$
C \lambda R(\lambda, A) R\left(\mu, A_{-1}\right) B v=\frac{\lambda}{\lambda-\mu} M R\left(\mu, A_{-1}\right) B v-\frac{1}{\lambda-\mu} M \lambda R\left(\lambda, A_{-1}\right) B v, \quad v \in U
$$

we have Range $\left(R\left(\mu, A_{-1}\right) B\right) \subset D\left(C_{\Lambda}\right)$ and

$$
C_{\Lambda} R\left(\mu, A_{-1}\right) B v=M R\left(\mu, A_{-1}\right) B v, \quad v \in U, \lambda \in \rho(A) .
$$

With these observations and using [12, lem.3.6], we have

$$
Z \subset D\left(C_{\Lambda}\right) \text { and }\left(C_{\Lambda}\right)_{\mid Z}=M \text {. }
$$

We also mention that (see [31]) if $(A, B, C)$ is regular then Range $\left(\Phi_{t}\right) \subset D\left(C_{\Lambda}\right)$ (and in particular the state of (2.15) $\left.z(t) \in D\left(C_{\Lambda}\right)\right)$ for a.e. $t \geq 0$. Moreover, $\left(\mathbb{F}_{\infty} u\right)(t)=C_{\Lambda} \Phi_{t} u$ for all $u \in L_{l o c}^{p}([0,+\infty), U)$ and a.e. $t \geq 0$. In addition, the observation function of the system (2.15) is given by

$$
y(t)=C_{\Lambda} z(t), \quad \text { a.e. } t>0
$$

for any initial condition $x \in X$ and any control function $u \in L_{l o c}^{p}([0,+\infty), U)$, see [31] for more details.

In many cases, it is very important to work not only with the linear operator $\mathbb{F}_{\infty}$ but also with its Laplace transform, called the transfer function. So if a system $(A, B, C)$ is regular then its transfer function if given by

$$
H(\lambda):=C_{\Lambda} R\left(\lambda, A_{-1}\right) B, \quad \operatorname{Re} \lambda>\omega_{0}(A) .
$$


According to Weiss [31], there exists $\gamma>0$ such that

$$
\sup _{\operatorname{Re\lambda >\gamma }}\|H(\lambda)\|<+\infty .
$$

In the rest of this section we shall present a perturbation theorem associated with regular linear systems, due to Weiss [31] in the Hilbert setting and Staffans [26, chap.7] for the Banach cases. To that purpose we need the following definition.

Definition 2.7. Let $(A, B, C)$ be a regular linear system on $X, U, U$. The identity operator $I_{U}: U \rightarrow U$ is called an admissible feedback if $I-\mathbb{F}_{\infty}$ has uniformly bounded inverse.

Theorem 2.8. Assume that the system $(A, B, C)$ is regular on $X, U, U$ and $I_{U}$ is an admissible feedback. Let $C_{\Lambda}$ be the Yosida extension of $C$ with respect to $A$. Then the operator

$$
\begin{aligned}
A^{c l} & :=A_{-1}+B C_{\Lambda} \\
D\left(A^{c l}\right) & :=\left\{x \in D\left(C_{\Lambda}\right):\left(A_{-1}+B C_{\Lambda}\right) x \in X\right\}
\end{aligned}
$$

generates a $C_{0}$-semigroup $\mathbb{T}^{c l}:=\left(T^{c l}(t)\right)_{t \geq 0}$ on $X$ such that

(i) Range $\left(T^{c l}(t)\right) \subset D\left(C_{\Lambda}\right)$ for a.e. $t>0$,

(ii) for any $x \in X$ and $t \geq 0$,

$$
T^{c l}(t) x=T(t) x+\int_{0}^{t} T_{-1}(t-s) B C_{\Lambda} T^{c l}(s) x d s,
$$

(iii) there exist constants $\alpha>0$ and $\delta_{\alpha}>0$ such that

$$
\int_{0}^{\alpha}\left\|C_{\Lambda} T^{c l}(t) x\right\|^{p} \leq \delta_{\alpha}^{p}\|x\|^{p}
$$

for any $x \in X$.

Let us denote by $X_{-1}^{A}$ (resp. $X_{-1}^{A^{c l}}$ ) be the extrapolation space associated with $X$ and $A$ (resp. $X$ and $A^{c l}$ ). Obviously these spaces are different. In [30], Weiss constructed subspaces $W_{A}$ and $W_{A^{c l}}$ of $X_{-1}^{A}$ and $X_{-1}^{A^{c l}}$, respectively, such that $J x:=\lim _{\lambda \rightarrow \infty} \lambda R\left(\lambda, A_{-1}\right) x$ in $X_{-1}^{A^{c l}}$ defines an isomorphism $J: W_{A} \longrightarrow W_{A^{c l}}$. Obviously, $J x=x$ in $X$. We obtain

$$
\int_{0}^{t} T_{-1}(t-s) B C_{\Lambda} T^{c l}(s) x d s=\int_{0}^{t} T_{-1}^{c l}(t-s) J B C_{\Lambda} T(s) x d s,
$$

see [30, p:54-55] and [25, Remark 4.6(b)] for more detail. Whence, from (2.24), the perturbed semigroup $\left(T^{c l}(t)\right)_{t \geq 0}$ satisfies also the following variation of constants formula

$$
T^{c l}(t) x=T(t) x+\int_{0}^{t} T_{-1}^{c l}(t-s) J B C_{\Lambda} T(s) x d s
$$

for any $t \geq 0$ and $x \in X$. 
Remark 2.9. Assume that the operator $M$ is linear bounded from $X$ to $U$. In this case, we take $C=M$ is an admissible operator for $A$ and $C_{\Lambda}=C=M$. In this case, the operator $\left(A^{c l}, D\left(A^{c l}\right)\right)$ is exactly the part of the operator $A_{-1}+B M$ in $X$. Moreover if ( $A, B)$ is well-posed, then the system $(A, B, M)$ is regular with $I_{U}$ is an admissible feedback operator. By applying Theorem 2.8 , the operator $\left(A^{c l}, D\left(A^{c l}\right)\right)$ generates a $C_{0}$-semigroup $\mathbb{T}^{c l}$ on $X$ such that

$$
T^{c l}(t) x=T(t) x+\int_{0}^{t} T_{-1}(t-s) B M T^{c l}(s) x d s,
$$

for any $t \geq 0$ and $x \in X$.

Remark 2.10. Consider the boundary value problem

$$
\begin{cases}\dot{z}(t)=A_{m} z(t), & t \geq 0, \\ G z(t)=M z(t), & t \geq 0, \\ z(0)=x, & \end{cases}
$$

where $A_{m}: Z \rightarrow X$ and $G, M: Z \rightarrow U$ as defined at the beginning of this section. The problem (2.27) can be viewed as a partial differential equation where the boundary operator $G$ is perturbed by another unbounded trace operator $M$. This system can also be reformulated as the following Cauchy problem in $X$,

$$
\left\{\begin{array}{l}
\dot{z}(t)=\mathcal{A} z(t), \quad t \geq 0, \\
z(0)=x,
\end{array}\right.
$$

where

$$
\mathcal{A}:=A_{m}, \quad D(\mathcal{A})=\{x \in Z, \quad G f=M f\} .
$$

Then the problem (2.27) is well-posed if the operator $(\mathcal{A}, D(\mathcal{A}))$ is a generator of a $C_{0^{-}}$ semigroup on $X$. Let the operator $A, B$ and $C$ defined by (2.1), (2.4) and (2.11), respectively. It is shown in [12] that if $(A, B, C)$ is regular and $I_{U}$ is admissible feedback then $\mathcal{A}$ coincides with the operator $A^{c l}$ defined by (2.22). Now according to Theorem 2.8, the operator $\mathcal{A}$ generates a $C_{0}$-semigroup $\mathcal{T}:=(\mathcal{T}(t))_{t \geq 0}$ on $X$ given by

$$
\mathcal{T}(t) x=T(t) x+\int_{0}^{t} T_{-1}(t-s) B C_{\Lambda} \mathcal{T}(s) x d s,
$$

for all $t \geq 0$ and $x \in X$. On the other hand it is shown in [12] that for any $\lambda \in \rho(A)$ we have

$$
\lambda \in \rho(\mathcal{A}) \Longleftrightarrow 1 \in \rho\left(D_{\lambda} M\right) \Longleftrightarrow 1 \in \rho\left(M D_{\lambda}\right) .
$$

Moreover, for $\lambda \in \rho(A) \cap \rho(\mathcal{A})$,

$$
R(\lambda, \mathcal{A})=\left(I-D_{\lambda} M\right)^{-1} R(\lambda, A) .
$$




\section{IMMEDIATE NORM CONTINUITY AND COMPACTNESS OF PERTURBED SEMIGROUPS}

In this section, we will work under assumptions of Theorem 2.8 (and also of Remark 2.10). We then suppose that semigroup $\mathbb{T}$ is norm continuity or compact and then show if the perturbed semigroup $\mathbb{T}^{c l}$ inherits such properties.

Let us first introduce a short proof of a result proved in [19] for Desch-Schappacher perturbations (i.e. in the case when the observation operator $C \in \mathcal{L}(X, U)$ in Theorem 2.8 or the boundary operator $M \in \mathcal{L}(X, U)$ in Remark 2.10). Before doing so, we recall from [9] the following characterization of immediately norm continuity for strongly continuous semigroups in Banach spaces.

Theorem 3.1. A strongly continuous semigroup $(T(t))_{t \geq 0}$ on a Banach $X$ is continuous in the operator norm for $t>0$ if and only if for all $\tau>0$ the operator

$$
K: L^{r}([0, \tau], X) \longrightarrow L^{r}([0, \tau], X), \quad(K f)(t)=\int_{0}^{t} T(t-s) f(s) d s
$$

satisfies the following Riesz condition $\left(R_{r}\right)$ for some (all) $r \in(1, \infty)$ i.e :

$$
\left(R_{r}\right) \quad \int_{0}^{\tau}\|(K f)(t+h)-(K f)(t)\|^{r} d t \rightarrow 0 \text { as } h \rightarrow 0
$$

uniformly for $f \in L^{r}([0, \tau], X)$ with $\|f\|_{r} \leq 1$.

We are now in the position to give a new proof [19, Theorem 6].

Theorem 3.2. Let the control system $(A, B)$ be well-posed on $X, U$ and $C \in \mathcal{L}(X, U)$. Assume that the semigroup $\mathbb{T}=(T(t))_{t \geq 0}$ is immediately norm continuous on $X$. Then the semigroup $\mathbb{T}^{c l}=\left(T^{c l}(t)\right)_{t \geq 0}$ is immediately norm continuous on $X$.

Proof. Let $0<h<\tau$ and $f \in L^{p}([0, \tau], X)$ with $\|f\|_{p} \leq 1$. We put

$$
\left(K^{c l} f\right)(t):=\int_{0}^{t} T^{c l}(t-s) f(s) d s
$$

We will prove that if $K$ satisfies $\left(R_{p}\right)$, then $K^{c l}$ also verifies $\left(R_{p}\right)$. In fact, By using (2.25), a change of variables and Fubini theorem, we obtain

$$
\left(K^{c l} f\right)(t)=(K f)(t)+\Phi_{t}^{J} C K f
$$

where we set

$$
\Phi_{t}^{J} v(\cdot)=\int_{0}^{t} T_{-1}^{c l}(t-s) J B v(s) d s, \quad t \geq 0, v \in L^{p}([0, t], U) .
$$

In view of (2.7) and (3.1),

$$
\begin{aligned}
\left(K^{c l} f\right)(t+h)-\left(K^{c l} f\right)(t) & =(K f)(t+h)-(K f)(t)+\Phi_{t+h}^{J} C K f-\Phi_{t}^{J} C K f \\
& =(K f)(t+h)-(K f)(t)+T^{c l}(t) \Phi_{h}^{J} C K f \\
& +\Phi_{t}^{J} C[(K f)(\cdot+h)-K f] .
\end{aligned}
$$


By admissibility of $J B$ for $\mathbb{T}^{c l}$, we obtain

$$
\begin{aligned}
\int_{0}^{\tau}\left\|\left(K^{c l} f\right)(t+h)-\left(K^{c l} f\right)(t)\right\|^{p} d t & \leq c_{p} \int_{0}^{\tau}\|(K f)(t+h)-(K f)(t)\|^{p} d t \\
& +c_{p} \int_{0}^{\tau}\left\|T^{c l}(t) \Phi_{h}^{J} C K f\right\|^{p} d t \\
& +c_{p} \int_{0}^{\tau}\left\|\Phi_{t}^{J} C[(K f)(\cdot+h)-K f]\right\|^{p} d t .
\end{aligned}
$$

Let $M \geq 1$ and $\tilde{\omega} \in \mathbb{R}$ such that $\left\|T^{c l}(t)\right\| \leq M e^{\tilde{\omega} t}$ for any $t \geq 0$ and $p>1$ such that $\frac{1}{p}+\frac{1}{q}=1$. By using (2.8),

$$
\begin{aligned}
\int_{0}^{\tau}\left\|T^{c l}(t) \Phi_{h}^{J} C(K f)(\cdot)\right\|^{p} d t & \leq M \tau e^{|\tilde{\omega}| \tau}\left\|\Phi_{h}^{J}\right\|^{p}\|C K f\|_{L^{p}([0, h], U)}^{p} \\
& \leq M \tau e^{|\tilde{\omega}| \tau}\left\|\Phi_{h}^{J}\right\|^{p} \beta h^{\frac{p}{q}}\|f\|_{p} \\
& \leq M \tau e^{|\tilde{\omega}| \tau}\left\|\Phi_{\tau}^{J}\right\|^{p} \beta h^{\frac{p}{q}}\|f\|_{p} \underset{h \rightarrow 0}{\longrightarrow} 0
\end{aligned}
$$

uniformly in $f$ such that $\|f\|_{p} \leq 1$, where $\beta$ is a constant independent of $f$. On the other hand, by using (2.8),

$$
\int_{0}^{\tau}\left\|\Phi_{t}^{J} C[(K f)(\cdot+h)-K f]\right\|^{p} d t \leq\left\|\Phi_{\tau}^{J}\right\|\|C\|^{p} \int_{0}^{\tau}\|(K f)(t+h)-(K f)(t)\|^{p} d t,
$$

which goes to 0 as $h \rightarrow 0$ uniformly in $\|f\|_{p} \leq 1$, due to the norm continuity of $\mathbb{T}$ and Theorem [3.1, respectively. This shows that

$$
\int_{0}^{\tau}\left\|\left(K^{c l} f\right)(t+h)-\left(K^{c l} f\right)(t)\right\|^{p} d t \underset{h \rightarrow 0}{\longrightarrow} 0
$$

uniformly in $\|f\|_{p} \leq 1$. Now according to Theorem $3.1, \mathbb{T}^{c l}$ is immediately norm continuous.

The following result on immediate norm continuity for perturbed semigroups can be considered as a generalization of a result proved in [19].

Theorem 3.3. Let assumptions of Theorem 2.8 be satisfied with $C$ is a zero-class admissible. In addition we suppose that the semigroup $\mathbb{T}=(T(t))_{t \geq 0}$ is immediately norm continuous on $X$. Then the perturbed semigroup $\mathbb{T}^{c l}=\left(T^{c l}(t)\right)_{t \geq 0}$ is immediately norm continuous on $X$ as well.

Proof. By assumption, for any $t>0$ we have

$$
\lim _{h \rightarrow 0}\|T(t+h)-T(t)\|=0 .
$$

Now let us prove that the perturbed semigroup $\mathbb{T}^{c l}$ introduced in Theorem 2.8 have also the above property. Due to (2.25), we have

$$
T^{c l}(t) x=T(t) x+R(t) x
$$


for all $t \geq 0$ and $x \in X$, where

$$
R(t) x=\int_{0}^{t} T_{-1}^{c l}(t-s) J B C_{\Lambda} T(s) x d s .
$$

According to (3.2), it suffices to prove the map $t \in(0, \infty) \mapsto R(t)$ is norm continuous. In fact, fix $t_{0}>0$ and choose arbitrary $h, \delta \in \mathbb{R}$ such that $0<|h|<\delta<t$. We then have

$$
\begin{aligned}
R(t+h) x- & R(t) x=\int_{0}^{\delta+h} T_{-1}^{c l}(t+h-s) J B C_{\Lambda} T(s) x d s+\int_{\delta+h}^{t+h} T_{-1}^{c l}(t+h-s) J B C_{\Lambda} T(s) x d s \\
& -\int_{0}^{\delta} T_{-1}^{c l}(t-s) J B C_{\Lambda} T(s) x d s-\int_{\delta}^{t} T_{-1}^{c l}(t-s) J B C_{\Lambda} T(s) x d s \\
& =T^{c l}(t-\delta)\left[\int_{0}^{\delta+h} T_{-1}^{c l}(\delta+h-s) J B C_{\Lambda} T(s) x d s-\int_{0}^{\delta} T_{-1}^{c l}(\delta-s) J B C_{\Lambda} T(s) x d s\right] \\
& +\int_{\delta}^{t} T_{-1}^{c l}(t-s) J B C_{\Lambda}[T(s+h) x-T(s) x] d s \\
& :=I_{1}(x, h, t)+I_{2}(x, h, t),
\end{aligned}
$$

where

$$
\begin{aligned}
& I_{1}(x, h, t):=T^{c l}(t-\delta)\left[\Phi_{\delta+h}^{J} C_{\Lambda} T(\cdot) x-\Phi_{\delta}^{J} C_{\Lambda} T(\cdot) x\right], \\
& I_{2}(x, h, t):=\int_{\delta}^{t} T_{-1}^{c l}(t-s) J B C_{\Lambda}[T(s+h) x-T(s) x] d s
\end{aligned}
$$

and

$$
\Phi_{\tau}^{J} v=\int_{0}^{\tau} T_{-1}^{c l}(\tau-s) J B v(s) d s, \quad \tau \geq 0, v \in L^{p}([0,+\infty), U) .
$$

By admissibility of $J B$ for $T^{c l}$, we have

$$
\left\|\Phi_{\tau}^{J} v\right\| \leq\left\|\Phi_{\tau}^{J}\right\|\|v\|_{L^{p}([0, \tau], U)}
$$

for any $\tau \geq 0$ and $v \in L^{p}([0,+\infty), U)$. Let $\tilde{M} \geq 1$ and $\omega \in \mathbb{R}$ such that $\left\|T^{c l}(t)\right\| \leq \tilde{M} e^{\omega t}$ for any $t \geq 0$. We estimate

$$
\begin{aligned}
\left\|I_{1}(x, h, t)\right\| & =\left\|T^{c l}(t-\delta)\left(\Phi_{\delta+h}^{J} C_{\Lambda} T(\cdot) x-\Phi_{\delta}^{J} C_{\Lambda} T(\cdot) x\right)\right\| \\
& \leq \tilde{M} e^{|\omega|(t-\delta)}\left(\left\|\Phi_{\delta+h}^{J}\right\|\left\|C_{\Lambda} T(\cdot) x\right\|_{L^{p}([0, \delta+h], U)}+\left\|\Phi_{\delta}^{J}\right\|\left\|C_{\Lambda} T(\cdot) x\right\|_{L^{p}([0, \delta], U)}\right) \\
& \leq 2 \tilde{M} e^{|\omega| t}\left\|\Phi_{2 \delta}^{J}\right\|\left\|C_{\Lambda} T(\cdot) x\right\|_{L^{p}([0,2 \delta], U)} \\
& \leq 2 \tilde{M} \gamma(2 \delta) e^{|\omega| t}\left\|\Phi_{2 \delta}^{J}\right\|\|x\|
\end{aligned}
$$

where $\gamma(2 \delta) \rightarrow 0$ as $\delta \rightarrow 0$, by (2.8) and admissibility of $C$ for $T$. We then have

$$
\left\|I_{1}(x, h, t)\right\| \leq \varpi_{t}(\delta)\|x\|
$$


for any $x \in X$, where $\varpi_{t}(\delta):=2 \tilde{M} \gamma(2 \delta) e^{|\omega| t}\left\|\Phi_{2 \delta}^{J}\right\| \rightarrow 0$ as $\delta \rightarrow 0$. By admissibility of $J B$ for $\mathbb{T}^{c l}$, a change of variables and admissibility of $C$ for $\mathbb{T}$, we obtain

$$
\begin{aligned}
\left\|I_{2}(x, h, t)\right\| & =\left\|\int_{0}^{t} T_{-1}^{c l}(t-s) J B C_{\Lambda}[T(s+h) x-T(s) x] \mathbb{I}_{[\delta, t]}(s) d s\right\| \\
& \leq\left\|\Phi_{t}^{J}\right\|\left(\int_{\delta}^{t}\left\|C_{\Lambda} T(s-\delta)[T(\delta+h) x-T(\delta) x]\right\|^{p} d s\right)^{1 / p} \\
& \leq\left\|\Phi_{t}^{J}\right\|\left(\int_{0}^{t-\delta}\left\|C_{\Lambda} T(s)[T(\delta+h) x-T(\delta) x]\right\|^{p}\right)^{1 / p} d s \\
& \leq\left\|\Phi_{t}^{J}\right\|\left(\int_{0}^{t}\left\|C_{\Lambda} T(s)[T(\delta+h) x-T(\delta) x]\right\|^{p} d s\right)^{1 / p} \\
& \leq \gamma(t)\left\|\Phi_{t}^{J}\right\|\|T(\delta+h) x-T(\delta) x\| .
\end{aligned}
$$

Combining (3.4), (3.6) and (3.7), we have

$$
\|R(t+h)-R(t)\| \leq \varpi_{t}(\delta)+\gamma(t)\left\|\Phi_{t}^{J}\right\|\|T(\delta+h)-T(\delta)\| .
$$

The fact that the semigroup $\mathbb{T}$ is immediately norm continuous implies that

$$
\lim _{h \rightarrow 0}\|R(t+h)-R(t)\| \leq \varpi_{t}(\delta) .
$$

By letting $\delta \rightarrow 0$, we obtain

$$
\lim _{h \rightarrow 0}\|R(t+h)-R(t)\|=0
$$

This ends the proof.

The next result is about immediate compactness of perturbed semigroups.

Theorem 3.4. Let assumptions of Theorem 2.8 be satisfied with $C$ is a zero-class admissible. In addition we suppose that the semigroup $\mathbb{T}=(T(t))_{t \geq 0}$ is immediately compact on $X$. Then perturbed semigroup $\mathbb{T}^{c l}=\left(T^{c l}(t)\right)_{t \geq 0}$ is immediately compact on $X$ as well.

Proof. According to (3.3) and the immediate compactness of the semigroup $\mathbb{T}$, it suffices to prove that the operators $R(t)$ are compact for any $t>0$. For this, we shall use an approximation argument. Take $\epsilon>0$ and define

$$
R_{\epsilon}(t) x=\int_{\epsilon}^{t} T_{-1}^{c l}(t-s) J B C_{\Lambda} T(s) x d s, \quad t>\varepsilon .
$$

We now show that $R_{\epsilon}(t)$ approaches to $R(t)$ uniformly as $\epsilon \rightarrow 0$. By admissibility of $J B$ for $\mathbb{T}^{c l}$ and $C$ for $\mathbb{T}$, we obtain

$$
\begin{aligned}
\left\|R(t) x-R_{\epsilon}(t) x\right\| & =\left\|T^{c l}(t-\epsilon) \int_{0}^{\epsilon} T_{-1}^{c l}(\epsilon-s) J B C_{\Lambda} T(s) x d s\right\| \\
& \leq\left\|T^{c l}(t-\epsilon)\right\|\left\|\Phi_{\epsilon}^{J}\right\|\left[\int_{0}^{\epsilon}\left\|C_{\Lambda} T(s) x\right\|^{p} d s\right]^{\frac{1}{p}} \\
& \leq \gamma(\epsilon)\left\|T^{c l}(t-\epsilon)\right\|\left\|\Phi_{\epsilon}^{J}\right\|\|x\| .
\end{aligned}
$$


This shows that $\left\|R(t)-R_{\epsilon}(t)\right\| \longrightarrow 0$ as $\epsilon \rightarrow 0$. Thus it suffices to show that $R_{\epsilon}(t)$ is compact for $t>\epsilon$. Let us consider a sequence $\left(x_{n}\right) \subset X$ with $\left\|x_{n}\right\| \leq 1$. Since $T(\epsilon)$ is compact, then there exists a subsequence $x_{\varphi(n)}$ such that

$$
T(\epsilon) x_{\varphi(n)} \longrightarrow y \in X \quad \text { as } n \rightarrow \infty .
$$

Hence

$$
\begin{aligned}
R_{\epsilon}(t) x_{\varphi(n)}= & \int_{\epsilon}^{t} T_{-1}^{c l}(t-s) J B C_{\Lambda} T(s-\epsilon) T(\epsilon) x_{\varphi(n)} d s \\
= & \int_{\epsilon}^{t} T_{-1}^{c l}(t-s) J B C_{\Lambda} T(s-\epsilon)\left[T(\epsilon) x_{\varphi(n)}-y\right] d s \\
& \quad+\int_{0}^{t-\epsilon} T_{-1}^{c l}(t-\epsilon-s) J B C_{\Lambda} T(s) y d s \\
:= & K_{\epsilon, t}\left(T(\epsilon) x_{\varphi(n)}-y\right)+R(t-\epsilon) y .
\end{aligned}
$$

Where

On the other hand,

$$
K_{\epsilon, t} x:=\int_{\epsilon}^{t} T_{-1}^{c l}(t-s) J B C_{\Lambda} T(s-\epsilon) x d s .
$$

$$
\begin{aligned}
\left\|K_{\epsilon, t}\left(T(\epsilon) x_{\varphi(n)}-y\right)\right\| & =\left\|\int_{0}^{t} T_{-1}^{c l}(t-s) J B C_{\Lambda} T(s-\epsilon)\left[T(\epsilon) x_{\varphi(n)}-y\right] \mathbb{I}_{[\epsilon, t]}(s) d s\right\| \\
& \leq\left\|\Phi_{t}^{J}\right\|\left[\int_{\epsilon}^{t}\left\|C_{\Lambda} T(s-\epsilon)\left[T(\epsilon) x_{\varphi(n)}-y\right]\right\|^{p} d s\right]^{\frac{1}{p}} \\
& \leq\left\|\Phi_{t}^{J}\right\|\left[\int_{0}^{t}\left\|C_{\Lambda} T(s)\left[T(\epsilon) x_{\varphi(n)}-y\right]\right\|^{p} d s\right]^{\frac{1}{p}} \\
& \leq \gamma(t)\left\|\Phi_{t}^{J}\right\|\left\|T(\epsilon) x_{\varphi(n)}-y\right\| \longrightarrow 0 \text { as } n \rightarrow \infty .
\end{aligned}
$$

Whence, $R_{\epsilon}(t) x_{\varphi(n)}$ converge to $R(t-\epsilon) y \in X$ which means that $R_{\epsilon}(t)$ are compact for $t>\epsilon$. This ends the proof.

Remark 3.5. As consequences of Theorem 3.3 and Theorem 3.4, we have

(1) If $(A, B)$ is well-posed (with $B \in \mathcal{L}\left(U, X_{-1}\right)$ ) and $C$ is bounded (i.e. $C \in \mathcal{L}(X, U)$ ), then assumptions of Theorem 2.8 are satisfied and $C$ is a zero class observation operator. Then $\mathbb{T}^{c l}$ is immediately norm continuous (resp. compact) whenever the semigroup $\mathbb{T}$ is.

(2) If $(A, C)$ is well-posed (with $C \in \mathcal{L}(D(A), U)$ ) and $B$ is bounded (i.e. $B \in$ $\mathcal{L}(U, X))$, then assumptions of Theorem 2.8 are satisfied. Let $\Phi_{\tau}^{J}$ as in the proof of Theorem 3.3. Using the boundedness of $B$ and Hölder inequality, we obtain

$$
\left\|\Phi_{\tau}^{J}\right\| \leq\|B\| \tau^{\frac{1}{q}}
$$

with $\frac{1}{p}+\frac{1}{q}=1$. Then by (3.5) we have $\left\|I_{1}(x, h, t)\right\| \rightarrow 0$ as $h \rightarrow 0$ uniformly in $x$. Then without assuming zero class property for $C$, we obtain that $\mathbb{T}^{c l}$ is immediately norm continuous (resp. compact) whenever the semigroup $\mathbb{T}$ is. 
The following theorem is a generalisation of a result proved in [17] in the case of Miyadera-Voigt perturbations.

Theorem 3.6. Let assumptions of Theorem 2.8 be satisfied with $C$ is a zero-class admissible. Then $R(t):=T^{c l}(t)-T(t)$ is compact for $t>0$ if and only if $R(t)$ is norm continuous for $t \geq 0$ and $R\left(\lambda, A^{c l}\right)-R(\lambda, A)$ is compact for $\lambda \in \rho\left(A^{c l}\right) \cap \rho(A)$.

Proof. The sufficient condition can be obtained by the same arguments as in [17]. Let us now prove the necessary conditions. The compactness of $R\left(\lambda, A^{c l}\right)-R(\lambda, A)$ is obtained by taking Laplace transform of compact operators $R(t)$ and using the result [21, theorem 3.3]. On the other hand for $t>0$ and $h$ near of zero, we have

$$
\begin{aligned}
R(t+h)-R(t) & =T^{c l}(t+h)-T(t+h)-R(t) \\
& =T^{c l}(h)\left(T^{c l}(t)-T(t)\right)+\left(T^{c l}(h)-T(h)\right) T(t)-R(t) \\
& =\left(T^{c l}(h)-I\right) R(t)+R(h) T(t) .
\end{aligned}
$$

Now the compactness of $R(t)$ implies that

$$
\left\|\left(T^{c l}(h)-I\right) R(t)\right\| \longrightarrow 0 \quad \text { as } h \rightarrow 0 .
$$

Moreover, using admissibility of $J B$ for $\mathbb{T}^{c l}$ and admissibility of $C$ for semigroup $\mathbb{T}$, we obtain

$$
\begin{aligned}
\|R(h) x\| & =\left\|\int_{0}^{h} T_{-1}^{c l}(h-s) J B C_{\Lambda} T(s) x d s\right\| \\
& \leq\left\|\Phi_{h}^{J}\right\|\left(\int_{0}^{h}\left\|C_{\Lambda} T(t) x\right\|^{p} d s\right)^{1 / p} \\
& \leq \gamma(h)\left\|\Phi_{h}^{J}\right\|\|x\|
\end{aligned}
$$

converges to 0 as $h \rightarrow 0$ for every $x \in X$. Hence,

$$
\|R(h)\| \longrightarrow 0 \quad \text { as } h \rightarrow 0 .
$$

This ends the proof.

Remark 3.7. In Theorems 3.3]3.4 and 3.6, we can replace the condition $C$ is zero class observation operator by a similar dual concept on admissible control operator $B$. In fact, let $B \in \mathcal{L}\left(U, X_{-1}\right)$ be an admissible control operator for $A$ with control maps $\Phi_{t}, t \geq 0$. For any $\tau>0$, there exists $c(\tau)>0$ such that

$$
\left\|\Phi_{\tau} u\right\| \leq c(\tau)\|u\|_{p}
$$

for all $u \in L^{p}\left(\mathbb{R}^{+}, U\right)$. Now we say that $B$ is a zero class control operator if the constant $c(\tau) \rightarrow 0$ as $\tau \rightarrow 0$. This notion is used in [14] to study input-to-state stability for the infinite-dimensional systems. Let assumptions of Theorem 2.8 be satisfied. From (2.24), we have

$$
\Phi_{\tau}^{J} C_{\Lambda} T(\cdot) x=\Phi_{\tau} C_{\Lambda} T^{c l}(\cdot) x
$$


for any $\tau \geq 0$ and $x \in X$. According to (3.11) and the admissibility of $C_{\Lambda}$ for $\mathbb{T}^{c l}$, there exists a constant $\tilde{\gamma}>0$ such that

$$
\left\|\Phi_{\tau}^{J} C_{\Lambda} T(\cdot) x\right\| \leq c(\tau) \tilde{\gamma}\|x\|
$$

Thus if in Theorems 3.35.4 and 3.6 instead of $C$ is a zero class observation operator we assume that $B$ is a zero class control operator, then we obtain the same results. In fact, in the proof of these theorems we replace the fact that $\gamma(\tau) \rightarrow 0$ as $\tau \rightarrow 0$ by $c(\tau) \rightarrow 0$ as $\rightarrow 0$, due to (3.5), (3.8), (3.11) and (3.12).

Example 3.8. Consider a one dimensional heat equation with mixed boundary conditions

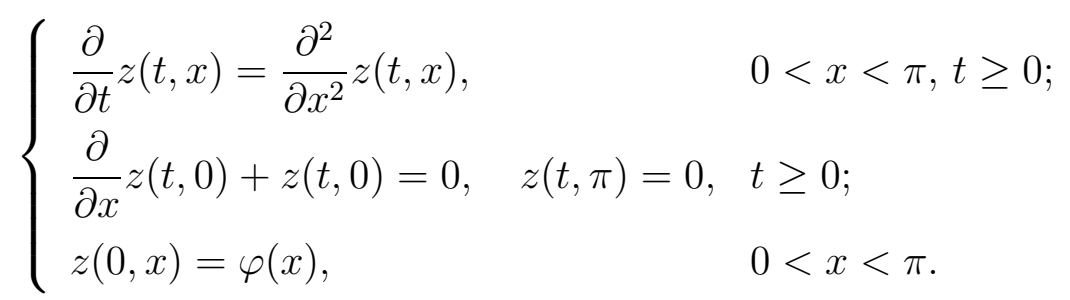

In order to use our abstract results, we select

$$
X:=L^{2}([0, \pi]), \quad Z:=\left\{f \in H^{2}([0, \pi]): f(\pi)=0\right\}, \quad \partial X:=\mathbb{C}
$$

and operators

$$
A_{m} f=f^{\prime \prime}, \quad G f=f^{\prime}(0) \quad \text { and } \quad M f=-f(0), \quad \text { for } \quad f \in Z .
$$

We know that the operator

$$
A \varphi:=A_{m} \varphi, \quad D(A)=\left\{f \in Z: f^{\prime}(0)=0\right\}
$$

generates an immediately norm continuous (even compact) $C_{0}$-semigroup $\mathbb{T}:=(T(t))_{t \geq 0}$ on $X$ (note that $A$ is self-adjoint). On the other hand, $G$ is surjective. So that the Dirichlet operator $D_{\lambda}$ exists for any $\lambda \in \rho(A)$, see the beginning of this section. As we are in the Hilbert setting, the extrapolation space $X_{-1}$ of $X$ associated with $A$ is isomorph to the topological dual space $\left(D\left(A^{*}\right)\right)^{\prime}$, where $A^{*}$ is the adjoint operator of $A$. We now put for any $\lambda \in \rho(A)$,

$$
B:=\left(\lambda-A_{-1}\right) D_{\lambda} \in \mathcal{L}\left(\mathbb{C}, D\left(A^{*}\right)^{\prime}\right)
$$

A straightforward computation shows that the adjoint operator of $B$ is given by

$$
B^{*} \varphi=-\varphi(0), \quad \varphi \in D\left(A^{*}\right)=D(A) .
$$

In addition, $B^{*}$ is an admissible observation operator for the adjoint semigroup $\mathbb{T}^{*}:=$ $\left(T^{*}(t)\right)_{t \geq 0}$. Hence, by duality, $B$ is an admissible control operator for the semigroup $\mathbb{T}$, (see [27, p.126]). In addition, $B^{*}$ is a zero-class observation operator (see [15, example $3.8])$. 
Moreover, by computation the Dirichlet operator is given by

$$
\begin{aligned}
& \left(D_{0} u\right)(x)=(x-\pi) \cdot u, \quad \text { for } \quad 0 \leq x \leq \pi \\
& \left(D_{\lambda} u\right)(x)=\frac{\sinh (\sqrt{\lambda}(x-\pi))}{\sqrt{\lambda} \cosh (\sqrt{\lambda} \pi)} \cdot u, \quad \text { for } \quad 0 \leq x \leq \pi \text { and } \lambda>0 .
\end{aligned}
$$

It is clear that Range $\left(D_{\lambda}\right) \subset Z$ and the transfer function $H(\lambda):=M D_{\lambda}$ is uniformly bounded on half plan. Consequently, $\left(A, B, B^{*}\right)$ generates a regular system on $L^{2}([0, \pi]), \mathbb{C}, \mathbb{C}$. It is well know that $A$ generates a compact semigroup in $X$. Hence by Theorem 3.4 the semigroup solution of heat equation with mixed boundary (3.13) is compact in $X$.

\section{The Eventual Differentiability under Desch-Schappacher PERTURBATIONS}

In this section, we still assume that assumptions of Theorem 2.8 are satisfied (hence the perturbed semigroup $\mathbb{T}^{c l}$ exists), and then discuss conditions for which there exists $\tau>0$ such that for any $x \in X$ the map $t \in(\tau, \infty) \rightarrow T^{c l}(t) x \in X$ is differentiable.

The following result due to Pazy [21, p.57] (see also [20]) gives a sufficient condition for differentiability of $C_{0}$-semigrpups on Banach spaces.

Theorem 4.1. Let $G: D(G) \subset X \rightarrow X$ be the generator of a $C_{0}$-semigroup $V:=$ $(V(t))_{t \geq 0}$ on $X$. If for some $\mu>\omega_{0}(G)$ we have

$$
\tau_{0}:=\limsup _{|\tau| \rightarrow+\infty} \log |\tau||| R(\mu+i \tau, G) \|<\infty .
$$

Then $V(t)$ is differentiable for $t>\tau_{0}$. In addition, the semigroup $V$ is immediately differentiable whenever $\tau_{0}=0$.

In the following result we generalize a Pazy result on the stability of differentiability under bounded perturbation (see [21]) to Desch-Schappacher perturbations operators.

Theorem 4.2. Let the control system $(A, B)$ be well-posed on $X, U$ and $C \in \mathcal{L}(X)$. Assume that the generator $(A, D(A))$ satisfies the condition (4.1). Then the assumption of Theorem 2.8 are satisfied and the perturbed semigroup $\mathbb{T}^{c l}$ is eventually differential from a time $2 \tau_{0}$. On the other hand, if $\tau_{0}=0$ then the semigroup $\mathbb{T}^{c l}$ is immediately differentiable.

Proof. According to Remark 2.9, the part of $A_{-1}+B C$ on $X$ denoted by $A^{\text {cl }}$ generates a strongly continuous semigroup $\mathbb{T}^{c l}$ given by the variation of constants formula (2.26). Taking Laplace transform on both sides of this formula we obtain

$$
R\left(\lambda, A^{c l}\right)=R(\lambda, A)+R\left(\lambda, A_{-1}\right) B C R\left(\lambda, A^{c l}\right), \quad \lambda \in \rho(A) .
$$


Let $\omega>\omega_{0}(A)$. According to (2.9) there exists a contants $c>0$ such that for any $\lambda \in \mathbb{C}$ with $\operatorname{Re} \lambda>\omega$,

$$
\left\|R\left(\lambda, A^{c l}\right)\right\|=\|R(\lambda, A)\|+\frac{c\|C\|}{(\operatorname{Re} \lambda-\omega)^{\frac{1}{p}}}\left\|R\left(\lambda, A^{c l}\right)\right\|
$$

Now, for $\mu>\omega+(2 c\|C\|)^{p}:=\omega^{\prime}$, and $\tau \in \mathbb{R}$, we obtain

$$
\left\|R\left(\mu+i \tau, A^{c l}\right)\right\| \leq 2\|R(\mu+i \tau, A)\| .
$$

Thus the result follows from Theorem 4.1.

Remark 4.3. Assume that $A: D(A) \subset X \rightarrow X$ is a generator of a $C_{0}$-semigroup $\mathbb{T}$ on $X, B \in \mathcal{L}(X)$ and $C \in \mathcal{L}(D(A), X)$ such that $(C, A)$ is well-posed. Then the condition of Theorem 2.8 are verified and the operator $A^{c l}=A+B C$ with domain $D\left(A^{c l}\right)=D(A)$, generates a $C_{0}$-semigroup $\mathbb{T}^{c l}$ on $X$ (see also [11]). Moreover,

$$
R\left(\lambda, A^{c l}\right)=R(\lambda, A)+R\left(\lambda, A^{c l}\right) B C R(\lambda, A), \quad \lambda \in \rho(A) .
$$

Let $\mu \in \mathbb{R}$ such that $\mu>\omega>\omega_{0}$ and $\tau \in \mathbb{R}$. According to (2.14), there exists a constant $c>0$ such that

$$
\left\|R\left(\mu+i \tau, A^{c l}\right)\right\| \leq\|R(\mu+i \tau, A)\|+\frac{c\|B\|}{(\mu-\omega)^{1-\frac{1}{p}}}\left\|R\left(\mu+i \tau, A^{c l}\right)\right\| .
$$

Now for $\mu>\omega+(2 c\|B\|)^{\frac{p}{p-1}}$, we have

$$
\left\|R\left(\mu+i \tau, A^{c l}\right)\right\| \leq 2\|R(\mu+i \tau, A)\| .
$$

As $A$ satisfies the condition (4.1), by Theorem 4.1, the generator of the perturbed semigroup $\mathbb{T}^{c l}$ satisfies also this condition and hence $\mathbb{T}^{c l}$ is a differential semigroup.

We also have the following observation about immediate norm continuity for perturbed semigroups on Hilbert spaces.

Remark 4.4. Assume that we work in the Hilbert setting and let us in the situations of Theorem 4.2 and/or Remark 4.3. In both cases we have proved that the estimate (4.2) for the generators $A$ and $A^{c l}$. It is well-known (see e.g. [7, p.115]) that $\mathbb{T}$ is immediately norm continuous on a Hilbert space $X$ if and only if $\|R(\mu+i \tau, A)\| \rightarrow 0$ as $\tau \rightarrow \pm \infty$. Now the inequality (4.2) shows that the immediate norm continuity is stable under Miyadera-Voigt and/or Desch-Schappacher perturbations.

\section{Application to Boundary integro-Differential EQUations}

let $X$ and $Z$ be Banach spaces with $Z \hookrightarrow X$ continuous and dense embedding. Let $A_{m}: Z \rightarrow X$ be a closed linear (differential) operator and an application $k: \mathbb{R}^{+} \rightarrow \mathbb{C}$ a measurable function. Consider the following boundary integro-differential equation

$$
\begin{cases}\dot{x}(t)=A_{m} x(t)+\int_{0}^{t} k(t-s) P x(s) d s, & t \geq 0 \\ G x(t)=M x(t), & t \geq 0, \\ x(0)=x, & \end{cases}
$$


where the initial condition $x \in X$ and boundary operators $G: Z \longrightarrow \partial X$ and $M: Z \longrightarrow$ $\partial X$ are linear.

The objective of this section is to study the well-posedness of the equation (5.1) and establish regularity of the solution. In the spirit of Greiner [10] and Salamon [24], we introduce the hypothesis

(H1) $A:=A_{m}$ with domain $D(A)=\operatorname{ker} G$ generates a $C_{0}$-semigroup $(T(t))_{t \geq 0}$ on $X$. (H2) The operator $G: Z \longrightarrow \partial X$ is surjective.

As discussed in the introductory section, let $D_{\lambda}$ be the Dirichlet operator associated with $A_{m}$ and $G$ and set $B:=\left(\lambda-A_{-1}\right) D_{\lambda}$ for $\lambda \in \rho(A)$. On the other hand, we select

$$
C:=M_{\mid D(A)}, \quad \text { and } \quad \mathbb{P}:=P_{\mid D(A)} .
$$

We further assume that

(H3) $(A, B, C)$ is a regular system on $X, \partial X, \partial X$ and $I: \partial X \longrightarrow \partial X$ is a feedback admissible with $C$ is a zero-class admissible.

(H4) $(A, B, \mathbb{P})$ is a regular system on $X, \partial X, X$.

In order to study the existence and regularity of the solution of the integro-differential equation (5.1), we need to introduce a Bergman space. Let then $h: \mathbb{R}^{+} \longrightarrow \mathbb{R}^{+}$be an admissible function (i.e $h$ is increasing, convex and $h(0)=0$ ). Hereafter, we assume that for $s>1$,

$$
\int_{0}^{1} h(\sigma)^{1-s} d \sigma<\infty
$$

Let $p, q \in(1,+\infty)$ be such that

$$
q=\frac{p s}{s-1}
$$

We define the sector

$$
\Sigma_{h}:=\{\sigma+i \tau \in \mathbb{C}, \sigma>0 \text { and }|\tau|<h(\sigma)\}
$$

The Bergman space is defined by

$$
\mathbb{B}_{h}^{q}\left(\Sigma_{h}, X\right):=\left\{f: \Sigma_{h} \rightarrow X \text { holomorphic such that }\|f\|_{\mathbb{B}_{h}^{q}\left(\Sigma_{h}, X\right)}<\infty\right\}
$$

with the norm

$$
\|f\|_{\mathbb{B}_{h}^{q}\left(\Sigma_{h}, X\right)}:=\left(\iint_{\Sigma_{h}}\|f(\sigma+i \tau)\|^{q} d \sigma d \tau\right)^{\frac{1}{q}}<\infty .
$$

We shall assume

(H5) $k(\cdot) \in \mathbb{B}_{h}^{q}\left(\Sigma_{h}, \mathbb{C}\right)$.

According to [4], the following translation semigroup on $\mathbb{B}_{h}^{q}\left(\Sigma_{h}, X\right)$,

$$
(S(t) f)(z):=f(t+z)
$$


with generator

$$
\frac{d}{d z} f=f^{\prime}, \quad D\left(\frac{d}{d z}\right):=\left\{f \in \mathbb{B}_{h}^{q}\left(\Sigma_{h}, X\right), f^{\prime} \in \mathbb{B}_{h}^{q}\left(\Sigma_{h}, X\right)\right\}
$$

is analytic. We first use product spaces to reformulate the equation (5.1) as abstract boundary value problem. In fact, consider the Banach space

$$
\mathcal{X}:=X \times \mathbb{B}_{h}^{q}\left(\Sigma_{h}, X\right) \quad \text { with norm } \quad\left\|\left(\begin{array}{l}
x \\
f
\end{array}\right)\right\|:=\|x\|+\|f\|_{\mathbb{B}_{h}^{q}\left(\Sigma_{h}, X\right)} .
$$

Moreover we consider the space

$$
\mathcal{Z}:=Z \times D\left(\frac{d}{d z}\right)
$$

The equation (5.1) can be rewritten as

$$
\begin{cases}\dot{z}(t)=\mathcal{A}_{m} z(t)+\mathcal{P} z(t), & t \geq 0 \\ \mathcal{G} z(t)=\mathcal{M} z(t), & t \geq 0, \\ z(0)=z \in \mathcal{X} & .\end{cases}
$$

where $\mathcal{A}_{m}, \mathcal{P}: \mathcal{Z} \rightarrow \mathcal{X}$ are given by

$$
\mathcal{A}_{m}:=\left(\begin{array}{cc}
A_{m} & 0 \\
0 & \frac{d}{d z}
\end{array}\right), \quad \mathcal{P}:=\left(\begin{array}{cc}
0 & \delta_{0} \\
k(\cdot) P & 0
\end{array}\right),
$$

and the boundary operators $\mathcal{G}, \mathcal{M}: \mathcal{Z} \rightarrow \partial X$ are defined by

$$
\mathcal{G}:=\left(\begin{array}{ll}
G & 0
\end{array}\right), \quad \mathcal{M}:=\left(\begin{array}{ll}
M & 0
\end{array}\right) .
$$

Now consider the operator

$$
\mathcal{A}:=\mathcal{A}_{m}, \quad D(\mathcal{A})=\{x \in Z: G x=M x\} \times D\left(\frac{d}{d z}\right) .
$$

The boundary problem (5.3) is reformulated again as a Cauchy problem

$$
\left\{\begin{array}{l}
\dot{z}(t)=\mathcal{A} z(t)+\mathcal{P} z(t), \quad t \geq 0 \\
z(0)=z
\end{array}\right.
$$

Lemma 5.1. Let assumptions (H1),(H2) and (H3) be satisfied. The the operator $(\mathcal{A}, D(\mathcal{A}))$ is the generator of a strongly continuous semigroup $(\mathcal{T}(t))_{t \geq 0}$ on $\mathcal{X}$, given by

$$
\mathcal{T}(t)=\left(\begin{array}{cc}
T^{c l}(t) & 0 \\
0 & S(t)
\end{array}\right), \quad t \geq 0,
$$

where $\left(T^{c l}(t)\right)_{t \geq 0}$ is the strongly continuous semigroup on $X$ generated by the operator

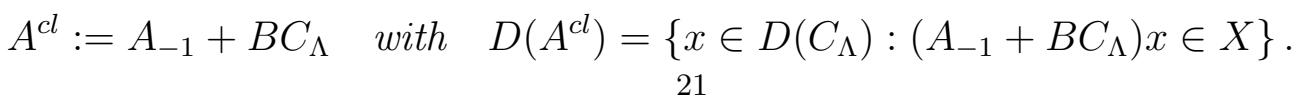


Proof. According to Remark 2.10, the following operator

$$
A^{c l}:=A_{m}, \quad D\left(A^{c l}\right)=\{x \in Z: G x=M x\}
$$

coincides with the operator defined by (5.5), which is a generator of a strongly continuous semigroup $\mathbb{T}^{c l}:=\left(T^{c l}(t)\right)_{t \geq 0}$, due to (H3) and Theorem 2.8. With these we have

$$
\mathcal{A}=\left(\begin{array}{cc}
A^{c l} & 0 \\
0 & \frac{d}{d z}
\end{array}\right), \quad D(\mathcal{A})=D\left(A^{c l}\right) \times D\left(\frac{d}{d z}\right) .
$$

This ends the proof.

Theorem 5.2. Let assumptions (H1) to (H5) be satisfied. Then the operator $(\mathcal{A}+$ $\mathcal{P}, D(\mathcal{A}))$ is a generator of a strongly continuous semigroup on $\mathcal{X}$.

Proof. According to [11], it suffices to prove that $\mathcal{P}$ is an admissible operator for $\mathcal{A}$. Let $\left(\begin{array}{l}x \\ f\end{array}\right) \in D(\mathcal{A})$. As $\mathcal{T}(t)\left(\begin{array}{l}x \\ f\end{array}\right) \in D(\mathcal{A})$, then

$$
T^{c l}(t) x \in D\left(A^{c l}\right) \quad \text { and } \quad S(t) f \in D\left(\frac{d}{d z}\right),
$$

for any $t \geq 0$. According to (2.20) and (5.2), we have

$$
\mathcal{P} \mathcal{T}(t)\left(\begin{array}{l}
x \\
f
\end{array}\right)=\left(\begin{array}{c}
f(t) \\
k(\cdot)) \mathbb{P}_{\Lambda} T^{c l}(t) x
\end{array}\right), \quad t \geq 0 .
$$

Here $\mathbb{P}_{\Lambda}$ is the Yosida extension of $\mathbb{P}$ relatively to $A$,. We recall that from feedback theory and the condition (H4), the operator $\mathbb{P}_{\Lambda}$ is an admissible observation operator for $\mathbb{T}^{c l}$. Then for constants $\lambda>0$ and $p>1$, there exist constants $\gamma>0$ and $c_{p}>0$ such that

$$
\begin{aligned}
\int_{0}^{\alpha}\left\|\mathcal{P} \mathcal{T}(t)\left(\begin{array}{l}
x \\
f
\end{array}\right)\right\|^{p} d t & \leq c_{p}\left(\int_{0}^{\alpha}\|f(t)\|_{X}^{p} d t+\int_{0}^{\alpha}\left\|k(\cdot) \mathbb{P}_{\Lambda} T^{c l}(t) x\right\|_{\mathbb{B}_{h}^{q}\left(\Sigma_{h}, X\right)}^{p} d t\right) \\
& \leq c_{p} \int_{0}^{\alpha}\|f(t)\|_{X}^{p} d t+\int_{0}^{\alpha}\left(\iint_{\Sigma_{h}}\left\|k(\sigma+i \tau) \mathbb{P}_{\Lambda} T^{c l}(t) x\right\|^{q} d \sigma d \tau\right)^{\frac{p}{q}} d t \\
& \leq c_{p} \int_{0}^{\alpha}\|f(t)\|_{X}^{p} d t+c_{p}\|k\|_{\mathbb{B}_{h}^{q}\left(\Sigma_{h}, \mathbb{C}\right)}^{p} \int_{0}^{\alpha}\left\|\mathbb{P}_{\Lambda} T^{c l}(t) x\right\|^{p} d t \\
& \leq c_{p} \int_{0}^{\alpha}\|f(t)\|_{X}^{p} d t+c_{p} \gamma^{p}\|k\|_{\mathbb{B}_{h}^{q}\left(\Sigma_{h}, \mathbb{C}\right)}^{p}\|x\|^{p} .
\end{aligned}
$$

On the other hand, using Cauchy formula, Jensen's inequality and similar arguments as in [5. Lem.4.3], one can see that there exists a constant $\kappa>0$ such that

$$
\int_{0}^{\alpha}\|f(t)\|_{X}^{p} d t \leq \kappa\|f\|_{\mathbb{B}_{h}^{q}\left(\Sigma_{h}, X\right)}^{p} .
$$

Now by taking $\vartheta:=c_{p} \max \left\{\kappa, \gamma^{p}\|k\|_{\mathbb{B}_{h}^{q}\left(\Sigma_{h}, \mathbb{C}\right)}^{p}\right\}$, we obtain

$$
\int_{0}^{\alpha}\left\|\mathcal{P} \mathcal{T}(t)\left(\begin{array}{l}
x \\
f
\end{array}\right)\right\|^{p} d t \leq \vartheta\left(\|x\|+\|f\|_{\mathbb{B}_{h}^{q}\left(\Sigma_{h}, X\right)}\right)^{p} .
$$

This ends the proof. 
Theorem 5.3. Let assumptions (H1) to (H5) be satisfied. Moreover, we assume that the operator A generates an immediately norm continuous semigroup on $X$. Then the operator $(\mathcal{A}+\mathcal{P}, D(\mathcal{A}))$ generates an immediately norm continuous semigroup on $\mathcal{X}$ as well.

Proof. Theorem 3.3 show that the operator $A^{c l}$ generates an immediately norm continuous semigroup $\mathbb{T}^{c l}$ on $X$. On the other hand, according [4], we know that the shift semigroup $S$ is analytic in the Bergman space $\mathbb{B}_{h}^{q}\left(\Sigma_{h}, X\right)$, hence it is immediately norm continuous semigroup. This show that the semigroup $(\mathcal{T}(t))_{t \geq 0}$ generated by $\mathcal{A}$ is immediately norm continuous. As $\mathcal{P}$ is a Miyadera-Voigt perturbation for $\mathcal{A}$, then by Remark 3.5 the operator $(\mathcal{A}+\mathcal{P}, D(\mathcal{A}))$ generates an immediately norm continuous semigroup on $\mathcal{X}$.

Theorem 5.4. Assume that $M \in \mathcal{L}(X, \partial X)$ and conditions (H1) to (H5) be satisfied. If for some $\mu>\omega_{0}(A)$ we have

$$
\tau_{0}:=\limsup _{|\tau| \rightarrow \infty} \log (|\tau|)\|R(\mu+i \tau, A)\|<\infty,
$$

then the semigroup generated by $(\mathcal{A}+\mathcal{P}, D(\mathcal{A}))$ is eventually differentiable.

Proof. As $A$ satisfies the Pazy condition (5.6), then by the proof of Theorem 4.2 the operator $A^{c l}$ satisfies also the Pazy condition. We know from [4] that the shift semigroup $(S(t))_{t \geq 0}$ is analytic on the Bergman space $\mathbb{B}_{h}^{q}\left(\Sigma_{h}, X\right)$. This implies that the operator $\mathcal{A}$ satisfies the Pazy condition. Now as $(\mathcal{P}, \mathcal{A})$ is admissible (see the proof of Theorem 5.2), then by Remark 4.3, the operator $(\mathcal{A}+\mathcal{P}, D(\mathcal{A}))$ satisfies also the Pazy condition. It follows from Theorem 4.1 that the operator $(\mathcal{A}+\mathcal{P}, D(\mathcal{A}))$ generates an eventually differentiable semigroup on $\mathcal{X}$.

Remark 5.5. In the case of $M \equiv 0$, the boundary integro-differential equation (5.1) becomes

$$
\left\{\begin{array}{l}
\dot{x}(t)=A x(t)+\int_{0}^{t} k(t-s) P x(s) d s, \quad t \geq 0, \\
x(0)=x \in X
\end{array}\right.
$$

In this case Bartà [5] showed the differentiability of the solutions by assuming a smooth regularity on the kernel $k$ that is $k^{\prime} \in \mathbb{B}_{h}^{q}\left(\Sigma_{h}, \mathbb{C}\right)$. However, in our results this extra condition on $k$ is not needed any more. On the other hand, the approach of Bartà is based on small perturbations, and cannot be extended to Desch-Schappacher perturbations. We think that our approach based on feedback theory of regular linear systems is the right way to solve such problems.

\section{REFERENCES}

[1] A. Bátkai and S. Piazzera, Semigroups and partial differential equations with delay, J. Math. Anal. Appl. 264 (2001) 1-20.

[2] C. J.K. Batty, Differentiability and growth bounds of solutions of delay equations, J. Math. Anal. Appl. 299 (2004) 133-146.

[3] C.J.K. Batty, S. Król, Perturbations of generators of $C_{0}$-semigroups and resolvent decay, J. Math. Anal. Appl. 367 (2010) 434-443. 
[4] T. Bárta, Analytic solutions of Volterra equations via semigroups, Semigroup Forum 76 (1) (2008) $142-148$.

[5] T. Bárta, Smooth solutions of Volterra equations via semigroups, Bulletin of the Australian Mathematical Society 78 (2008) 249-260.

[6] Bogdan D. Doytchinov, W.J . Hrusa and S.J . Watson, On Perturbations of Differentiable Semigroups, Semigroup Forum 54 (1997) 100-111.

[7] K. J. Engel and R. Nagel, One-parameter Semigroups for Linear Systems, Springer-Verlag, New York, 2000.

[8] H. O. Fattorini, Boundary control systems, SIAM J. Control 22 (1968) 349-385.

[9] V. Goersmeyer, L. Weis, Norm continuity of $C_{0}$-semigroups, Studia Math. 134 (2) (1999) 169-178.

[10] G. Greiner, Perturbing the boundary conditions of a generator, Houston J. Math. 13 (1987) 213-229.

[11] S. Hadd, Unbounded perturbation of $C_{0}$-semigroups on Bananch spaces and applications, Semigroup Forum 70 (2005) 451-465.

[12] S. Hadd, R. Manzo and A. Rhandi, Unbounded perturbations of the Generator domain, Discrete and Continuous Dynamical Systems 35 (2015) 703-723.

[13] P.S. Iley, Perturbations of differentiable semigroups, J. Evol. Equ. 7 (2007) 765-781.

[14] B. Jacob, R. Nabiulin, J.R. Partington and F.L. Schwenninger, Infinite-dimentional input-to-state stability and Orlicz spaces, SIAM J. Control Optim. 56 (2) (2018) 868-889.

[15] B. Jacob, J.R. Partington, S. Pott, Zero-class admissibility of observation operators, Systems Control Lett. 58 (2009) 406-412.

[16] M. Jung, Multiplicative Perturbations in Semigroup Theory With the (Z)-Condition, Semigroup Forum 52 (1996) 197-211.

[17] M. Li, X. Gu and F. Huang, On Unbounded Perturbations of Semigroups: Compactness and Norm Continuity, Semigroup Forum 65 (2002) 58-70.

[18] T. Mátrai, On Perturbations of Eventually Compact Semigroups Preserving Eventual Compactness, Semigroup Forum 69 (2004) 317-340.

[19] T. Mátrai, On perturbations preserving the immediate norm continuity of semigroups, J. Math. Anal. Appl. 341 (2008) 961-974.

[20] A. Pazy, On the differentiability and compactness of semigroups of linear operators, J. Math. Mech 17 (1968) 1131-1141.

[21] A. Pazy, Semigroups of Linear Operators and Applications to Partial Differential Equations, Springer-Verlag, Berlin, 1983.

[22] R. S. Phillips, Perturbation theory for semigroups of linear operators, Trans. Amer. Math. Soc. 74 (1953) 199-221

[23] M. Renardy, On the stability of differentiability of semigroups, Semigroup Forum 51 (1995) 343-346.

[24] D. Salamon, Infinite-dimensional linear system with unbounded control and observation: a functional analytic approach, Trans. Amer. Math. Soc. 300 (1987) 383-431.

[25] R. Schnaublet, Feedbacks for nonautonomous regular linear systems, SIAM J. Control Optim. 41 (4) (2002) 1141-1165.

[26] O.J. Staffans, Well-posed Linear Systems, Encyclopedia of Mathematics and its Applications, 103, Cambridge University Press, Cambridge, 2005.

[27] M. Tucsnak and G. Weiss, Observation and Control for Operator Semigroups, Birkhäser, Basel, Boston, Berlin, 2009.

[28] G. Weiss, Admissible observation operators for linear semigroups, Israel J. Math. 65 (1989) 17-43.

[29] G. Weiss, Admissibility of unbounded control operators, SIAM J. Control Optim. 27 (1989) 527-545.

[30] G. Weiss, Regular linear systems with feedback, Math. Control signals Systems 7 (1994) 23-57.

[31] G. Weiss, Transfer functions of regular linear systems. I. Characterization of regularity, Trans. Amer. Math. Soc. 342 (1994) 827-854. 
[32] L. Zhang, Perturbations of eventually differentiable and eventually norm-continuous semigroups on Banach spaces, J. Math. Anal. Appl. 322 (2006) 523-529.

[33] G.Q. Xu, C. Liu and S.P. Yung, Necessary conditions for the exact observability of systems on Hilbert spaces, Systems Control Letters 57 (2008) 222-227.

Department of Mathematics, Faculty of Sciences, Ibn Zohr University, Hay Dakhla, BP8106, 80000-AGAdir, MorocCo; ABED.Boulouz@EDu.uiz.AC.MA, H.BOUNiT@Uiz.AC.MA, A.DRIOUICH@UIZ.AC.MA, S.HADD@UIZ.AC.MA 